\title{
A generalized statistical convergence in intuitionistic fuzzy normed spaces
}

\author{
Ekrem Savaş $^{\mathrm{a}}$, Mehmet Gürdal ${ }^{\mathrm{b}, *}$ \\ a Department of Mathematics, Istanbul Ticaret University, Üsküdar-Istanbul, Turkey \\ b Suleyman Demirel University, Department of Mathematics, 32260, Isparta, Turkey \\ *Corresponding author, e-mail: gurdalmehmet@sdu.edu.tr
}

Received 2 Feb 2015

Accepted 22 Jul 2015

\begin{abstract}
In this paper, we introduce the notion of $\mathscr{I}$ - $[V, \lambda]$-summability and $\mathscr{I}$ - $\lambda$-statistical convergence with respect to the intuitionistic fuzzy norm $(\mu, v)$, investigate their relationship, and make some observations about these classes. We mainly examine the relation between these two new methods and the relation between $\mathscr{I}$ - $\lambda$-statistical convergence and $\mathscr{I}$-statistical convergence in the corresponding intuitionistic fuzzy normed space.
\end{abstract}

KEYWORDS: ideal, filter, $\mathscr{I}$-statistical convergence, $\mathscr{I}$ - $\lambda$-statistical convergence, $\mathscr{I}$-[V, $\lambda]$-summability

MSC2010: 40G99

\section{INTRODUCTION AND PRELIMINARIES}

The idea of convergence of a real sequence has been extended to statistical convergence by Fast ${ }^{1}$ as follows: let $K$ be a subset of $\mathbb{N} \equiv\{1,2, \ldots\}$. Then the asymptotic density of $K$ is defined by $\delta(K):=$ $\lim _{n \rightarrow \infty}(1 / n)|\{k \leqslant n: k \in K\}|$, where $|S|$ denotes the cardinality of the set $S$. A number sequence $x=\left(x_{k}\right)_{k \in \mathbb{N}}$ is said to be statistically convergent to $L$ if for every $\varepsilon>0, \delta\left(\left\{k \in \mathbb{N}:\left|x_{k}-L\right| \geqslant \varepsilon\right\}\right)=0$. If $\left(x_{k}\right)_{k \in \mathbb{N}}$ is statistically convergent to $L$ we write $s t-\lim x_{k}=L$. Statistical convergence turned out to be one of the most active areas of research in summability theory after the work of Fridy ${ }^{2}$ and Šalát ${ }^{3}$.

In Ref. 4, Kostyrko et al introduced the concept of $\mathscr{I}$-convergence of sequences in a metric space and studied some properties of such convergence. Note that $\mathscr{I}$-convergence is an interesting generalization of statistical convergence. More investigations in this direction and more applications of ideals can be found in Refs. 5-7. In another direction, the idea of $\lambda$-statistical convergence was introduced and studied by Mursaleen ${ }^{8}$ as an extension of the $[V, \lambda]$ summability of Leindler ${ }^{9}$. $\lambda$-statistical convergence is a special case of the more general A-statistical convergence studied in Ref. 10.

Following the introduction of fuzzy set theory by Zadeh ${ }^{11}$, there has been extensive research to find applications and fuzzy analogues of the classical theories. The theory of intuitionistic fuzzy sets was introduced by Atanassov ${ }^{12}$; it has been extensively used in decision-making problems ${ }^{13}$. The concept of an intuitionistic fuzzy metric space was introduced in Ref. 14. Saadati and Park ${ }^{15}$ introduced the notion of an intuitionistic fuzzy normed space. Some work related to the convergence of sequences in several normed linear spaces in a fuzzy setting can be found in Refs. 16-19.

Here we intend to unify these two approaches and use ideals to introduce the concept of $\mathscr{I}-\lambda$ statistical convergence with respect to the intuitionistic fuzzy norm $(\mu, v)$, and investigate some of its consequences.

Definition 1 [Ref. 20] A triangular norm ( $t$-norm) is a continuous mapping $*:[0,1] \times[0,1] \rightarrow[0,1]$ such that $(S, *)$ is an abelian monoid with unit one and $c * d \leqslant a * b$ if $c \leqslant a$ and $d \leqslant b$ for all $a, b, c, d \in$ $[0,1]$.

Definition 2 [Ref. 20] A binary operation $\diamond$ : $[0,1] \times[0,1] \rightarrow[0,1]$ is said to be a continuous $t$ conorm if it satisfies the following conditions:

(i) $\diamond$ is associative and commutative,

(ii) $\diamond$ is continuous,

(iii) $a \diamond 0=a$ for all $a \in[0,1]$,

(iv) $a \diamond b \leqslant c \diamond d$ whenever $a \leqslant c$ and $b \leqslant d$ for each $a, b, c, d \in[0,1]$.

For example, we can give $a * b=a b, a *$ $b=\min \{a, b\}, a \diamond b=\min \{a+b, 1\}$ and $a \diamond b=$ $\max \{a, b\}$ for all $a, b \in[0,1]$. 
Using the continuous $t$-norm and $t$-conorm, Saadati and Park ${ }^{15}$ has recently introduced the concept of intuitionistic fuzzy normed space as follows.

Definition 3 [Ref. 15] The five-tuple $(X, \mu, \nu, *, \diamond)$ is said to be an intuitionistic fuzzy normed space (for short, IFNS) if $X$ is a vector space, $*$ is a continuous $t$-norm, $\diamond$ is a continuous $t$-conorm, and $\mu, v$ are fuzzy sets on $X \times(0, \infty)$ satisfying the following conditions for every $x, y \in X$, and $s, t>0$ :

(i) $\mu(x, t)+v(x, t) \leqslant 1$;

(ii) $\mu(x, t)>0$;

(iii) $\mu(x, t)=1$ if and only if $x=0$;

(iv) $\mu(\alpha x, t)=\mu(x, t /|\alpha|)$ for each $\alpha \neq 0$;

(v) $\mu(x, t) * \mu(y, s) \leqslant \mu(x+y, t+s)$;

(vi) $\mu(x, \cdot):(0, \infty) \rightarrow[0,1]$ is continuous;

(vii) $\lim _{t \rightarrow \infty} \mu(x, t)=1$ and $\lim _{t \rightarrow 0} \mu(x, t)=0$;

(viii) $v(x, t)<1$;

(ix) $v(x, t)=0$ if and only if $x=0$;

(x) $v(\alpha x, t)=\mu(x, t /|\alpha|)$ for each $\alpha \neq 0$;

(xi) $v(x, t) \diamond v(y, s) \geqslant v(x+y, t+s)$;

(xii) $v(x, \cdot):(0, \infty) \rightarrow[0,1]$ is continuous;

(xiii) $\lim _{t \rightarrow \infty} v(x, t)=0$ and $\lim _{t \rightarrow 0} v(x, t)=1$.

In this case $(\mu, v)$ is called an intuitionistic fuzzy norm. As a standard example, we can give the following. Let $(X,\|\cdot\|)$ be a normed space, and let $a * b=a b$ and $a \diamond b=\min \{a+b, 1\}$ for all $a, b \in$ $[0,1]$. For all $x \in X$ and every $t>0$, consider

$$
\mu(x, t)=\frac{t}{t+\|x\|} \text { and } v(x, t)=\frac{\|x\|}{t+\|x\|} .
$$

Then observe that $(X, \mu, v, *, \diamond)$ is an intuitionistic fuzzy normed space.

Definition 4 [Ref. 15] Let $(X, \mu, v, *, \diamond)$ be an IFNS. Then a sequence $x=\left\{x_{k}\right\}$ is said to be convergent to $L \in X$ with respect to the intuitionistic fuzzy norm $(\mu, v)$ if, for every $\varepsilon>0$ and $t>0$, there exists $k_{0} \in \mathbb{N}$ such that $\mu\left(x_{k}-L, t\right)>1-\varepsilon$ and $v\left(x_{k}-L, t\right)<\varepsilon$ for all $k \geqslant k_{0}$. It is denoted by

$$
(\mu, v)-\lim x=L \text { or } x_{k} \stackrel{(\mu, v)}{\rightarrow} L
$$

as $k \rightarrow \infty$.

\section{$\mathscr{I}$ - $\lambda$-STATISTICAL CONVERGENCE ON IFNS}

In this section we deal with the relation between these two new methods and with relations between $\mathscr{I}$ - $\lambda$-statistical convergence and $\mathscr{I}$-statistical convergence introduced by the authors recently in an intuitionistic fuzzy normed space. Before proceeding further, we should recall some notation for $\mathscr{I}$ statistical convergence and ideal convergence.
The family $\mathscr{I} \subset 2^{Y}$ of subsets of a nonempty set $Y$ is said to be an ideal in $Y$ if (i) $\varnothing \notin \mathscr{I}$; (ii) $A, B \in \mathscr{I}$ imply $A \cup B \in \mathscr{I}$; (iii) $A \in \mathscr{I}, B \subset A$ imply $B \in \mathscr{I}$, while an admissible ideal $\mathscr{I}$ of $Y$ further satisfies $\{x\} \in \mathscr{I}$ for each $x \in Y$. If $\mathscr{I}$ is an ideal in $Y$ then the collection $F(\mathscr{I})=\left\{M \subset Y: M^{c} \in \mathscr{I}\right\}$ forms a filter in $Y$ which is called the filter associated with $\mathscr{I}$. Let $\mathscr{I} \subset 2^{\mathbb{N}}$ be a nontrivial ideal in $\mathbb{N}$. Then a sequence $\left\{x_{n}\right\}_{n \in \mathbb{N}}$ in $X$ is said to be $\mathscr{I}$-convergent to $x \in X$, if for each $\varepsilon>0$ the set $A(\varepsilon)=\left\{n \in \mathbb{N}:\left\|x_{n}-x\right\| \geqslant \varepsilon\right\}$ belongs to $\mathscr{I}$ (see Ref. 4).

Definition 5 [Refs. 6,7] A sequence $x=\left\{x_{k}\right\}_{k \in \mathbb{N}}$ is said to be $\mathscr{I}$-statistically convergent to $L$ or $S(I)$ convergent to $L$ if, for each $\varepsilon>0$ and $\delta>0$,

$$
\left\{n \in \mathbb{N}: \frac{1}{n}\left|\left\{k \leqslant n:\left\|x_{k}-L\right\| \geqslant \varepsilon\right\}\right| \geqslant \delta\right\} \in \mathscr{I}
$$

or equivalently if for each $\varepsilon>0$

$$
\delta_{\mathscr{I}}(A(\varepsilon))=\mathscr{I}-\lim \delta_{n}(A(\varepsilon))=0,
$$

where $A(\varepsilon)=\left\{k \leqslant n:\left\|x_{k}-L\right\| \geqslant \varepsilon\right\}$ and $\delta_{n}(A(\varepsilon))=$ $|A(\varepsilon)| / n$.

In this case we write $x_{k} \rightarrow L(S(\mathscr{I}))$. The class of all $\mathscr{I}$-statistically convergent sequences will be denoted simply by $S(\mathscr{I})$. Let $\mathscr{I}_{\mathrm{f}}$ be the family of all finite subsets of $\mathbb{N}$. Then $\mathscr{I}_{\mathrm{f}}$ is an admissible ideal in $\mathbb{N}$ and $\mathscr{I}$-statistical convergence is the statistical convergence.

Definition 6 [Ref. 21] Let $(X, \mu, v, *, \diamond)$ be an IFNS. Then a sequence $x=\left(x_{k}\right)$ is said to be $\mathscr{I}$ statistically convergent to $L \in X$ with respect to the intuitionistic fuzzy normed space and is denoted by

$$
x_{k} \stackrel{(\mu, v)}{\rightarrow} L\left(S^{(\mu, v)}(\mathscr{I})\right),
$$

if for every $\varepsilon>0$, and every $\delta>0$ and $t>0$,

$$
\begin{gathered}
\left\{n \in \mathbb{N}: \frac{1}{n} \mid\left\{k \leqslant n: \mu\left(x_{k}-L, t\right) \leqslant 1-\varepsilon\right.\right. \\
\text { or } \left.\left.v\left(x_{k}-L, t\right) \geqslant \varepsilon\right\} \mid \geqslant \delta\right\} \in \mathscr{I} .
\end{gathered}
$$

Let $\mathscr{I}_{\mathrm{f}}$ be the family of all finite subsets of $\mathbb{N}$. Then $\mathscr{I}_{\mathrm{f}}$ is an admissible ideal in $\mathbb{N}$, and $\mathscr{I}$-statistical convergence coincides with the notion of statistical convergence introduced in Ref. 22.

Let $\lambda=\left(\lambda_{n}\right)$ be a non-decreasing sequence of positive numbers tending to $\infty$ such that $\lambda_{n+1} \leqslant$ $\lambda_{n}+1, \lambda_{1}=1$. The collection of such a sequence $\lambda$ will be denoted by $\Delta$. 
The generalized de Valée-Pousin mean is defined by

$$
t_{n}(x)=\frac{1}{\lambda_{n}} \sum_{k \in I_{n}} x_{k}
$$

where $I_{n}=\left[n-\lambda_{n}+1, n\right]$. We are now ready to obtain our main results.

Definition 7 Let $(X, \mu, v, *, \diamond)$ be an IFNS. A sequence $x=\left(x_{k}\right)$ is said to be $\mathscr{I}$-[V, $\left.\lambda\right]$-summable to $L \in X$ with respect to the intuitionistic fuzzy norm $(\mu, v)$ and is denoted by $\mathscr{I}-[V, \lambda]^{(\mu, v)}-\lim x=L$, if for any $\delta>0$ and $t>0$,

$$
\begin{gathered}
\left\{n \in \mathbb{N}: \mu\left(t_{n}(x)-L, t\right) \leqslant 1-\delta\right. \\
\left.\quad \text { or } v\left(t_{n}(x)-L, t\right) \geqslant \delta\right\} \in \mathscr{I} .
\end{gathered}
$$

Definition 8 A sequence $x=\left(x_{k}\right)$ is said to be $\mathscr{I}$ $\lambda$-statistically convergent or $\mathscr{I}-S_{\lambda}$ convergent to $L$ with respect to the intuitionistic fuzzy norm $(\mu, v)$, and denoted by $\mathscr{I}-S_{\lambda}^{(\mu, v)}-\lim x=L$ or $x_{k} \rightarrow L(\mathscr{I}$ $S_{\lambda}^{(\mu, v)}$ ), if for every $\varepsilon>0, \delta>0$ and $t>0$,

$$
\begin{gathered}
\left\{n \in \mathbb{N}: \frac{1}{\lambda_{n}} \mid\left\{k \in I_{n}: \mu\left(x_{k}-L, t\right) \leqslant 1-\varepsilon\right.\right. \\
\text { or } \left.\left.v\left(x_{k}-L, t\right) \geqslant \varepsilon\right\} \mid \geqslant \delta\right\} \in \mathscr{I} .
\end{gathered}
$$

Let $\mathscr{I}_{\mathrm{f}}$ be the family of all finite subsets of $\mathbb{N}$. Then $\mathscr{I}_{\mathrm{f}}$ is an admissible ideal in $\mathbb{N}$ and $\mathscr{I}$ $\lambda$-statistical convergence is the $\lambda$-statistical convergence introduced in Ref. 8.

We shall denote by $S^{(\mu, v)}(\mathscr{I}), S_{\lambda}^{(\mu, v)}(\mathscr{I})$ and $[V, \lambda]^{(\mu, v)}(\mathscr{I})$ the collections of all $\mathscr{I}$-statistically convergent, $\mathscr{I}-S_{\lambda}^{(\mu, v)}$-convergent and $\mathscr{I}$ - $[V, \lambda]^{(\mu, v)}$ convergent sequences, respectively.

Theorem 1 Let $(X, \mu, v, *, \diamond)$ be an IFNS, and let $\lambda=$ $\left(\lambda_{n}\right)$ be a sequence in $\Delta$.

(i) If $x_{n} \rightarrow L[V, \lambda]^{(\mu, v)}(\mathscr{I})$ then $x_{k} \rightarrow L\left(S_{\lambda}^{(\mu, v)}(\mathscr{I})\right)$.

(ii) If $x \in m(X)$, the space of all bounded sequences of $X$ and $x_{k} \rightarrow L\left(S_{\lambda}^{(\mu, v)}(\mathscr{I})\right)$ then $x_{k} \rightarrow$ $L[V, \lambda]^{(\mu, \nu)}(\mathscr{I})$.

(iii) $S_{\lambda}^{(\mu, \nu)}(\mathscr{I}) \cap m(X)=[V, \lambda]^{(\mu, v)}(\mathscr{I}) \cap m(X)$.

Proof: (i) By hypothesis, for every $\varepsilon>0, \delta>0$ and $t>0$, let $x_{k} \rightarrow L[V, \lambda]^{(\mu, v)}(\mathscr{I})$. We have

$$
\begin{gathered}
\sum_{k \in I_{n}}\left(\mu\left(x_{k}-L, t\right) \text { or } v\left(x_{k}-L, t\right)\right) \\
\geqslant \sum_{\substack{k \in I_{n} \& \mu\left(x_{k}-L, t\right)<1-\varepsilon \\
\text { or } v\left(x_{k}-L, t\right)>\varepsilon}}\left(\mu\left(x_{k}-L, t\right) \text { or } v\left(x_{k}-L, t\right)\right) \\
\geqslant \varepsilon \mid\left\{k \in I_{\mathrm{r}}: \mu\left(x_{k}-L, t\right) \leqslant 1-\varepsilon\right. \text { or } \\
\left.v\left(x_{k}-L, t\right) \geqslant \varepsilon\right\} \mid .
\end{gathered}
$$

Then observe that

$$
\begin{gathered}
\frac{1}{\lambda_{n}} \mid\left\{k \in I_{n}: \mu\left(x_{k}-L, t\right) \leqslant 1-\varepsilon\right. \text { or } \\
\left.v\left(x_{k}-L, t\right) \geqslant \varepsilon\right\} \mid \geqslant \delta \\
\Rightarrow \frac{1}{\lambda_{n}} \sum_{k \in I_{n}} \mu\left(x_{k}-L, t\right) \leqslant(1-\varepsilon) \delta \text { or } \\
\frac{1}{\lambda_{n}} \sum_{k \in I_{n}} v\left(x_{k}-L, t\right) \geqslant \varepsilon \delta
\end{gathered}
$$

which implies

$$
\begin{gathered}
\left\{n \in \mathbb{N}: \frac{1}{\lambda_{n}} \mid\left\{k \in I_{n}: \mu\left(x_{k}-L, t\right) \leqslant 1-\varepsilon\right. \text { or }\right. \\
\left.\left.v\left(x_{k}-L, t\right) \geqslant \varepsilon\right\} \mid \geqslant \delta\right\} \\
\subset\left\{n \in \mathbb{N}: \frac{1}{\lambda_{n}}\left\{\sum_{k \in I_{n}} \mu\left(x_{k}-L, t\right) \leqslant 1-\varepsilon\right. \text { or }\right. \\
\left.\left.\sum_{k \in I_{n}} v\left(x_{k}-L, t\right) \geqslant \varepsilon\right\} \geqslant \varepsilon \delta\right\} .
\end{gathered}
$$

Since $x_{k} \rightarrow L[V, \lambda]^{(\mu, v)}(\mathscr{I})$, we immediately see that $x_{k} \rightarrow L\left(S_{\lambda}^{(\mu, v)}\right)$, whence the result.

(ii) We assume that $x_{k} \rightarrow L\left(S_{\lambda}^{(\mu, v)}(\mathscr{I})\right)$ and $x \in$ $l_{\infty}^{(\mu, v)}$. The inequalities $\mu\left(x_{k}-L, t\right) \geqslant 1-M$ or $v\left(x_{k}-\right.$ $L, t) \leqslant M$ hold for all $k$. Let $\varepsilon>0$ be given. Then we have

$$
\begin{aligned}
& \frac{1}{\lambda_{n}} \sum_{k \in I_{n}}\left(\mu\left(x_{k}-L, t\right) \text { or } v\left(x_{k}-L, t\right)\right) \\
& =\frac{1}{\lambda_{n_{n}}} \sum_{\substack{k \in I_{n} \\
\& \mu\left(x_{k}-L, t\right) \leqslant 1-\varepsilon \\
v\left(x_{k}-L, t\right) \geqslant \varepsilon}}\left(\mu\left(x_{k}-L, t\right) \operatorname{or} v\left(x_{k}-L, t\right)\right) \\
& \quad+\frac{1}{\lambda_{n}} \sum_{\substack { k \in I_{n} \\
\begin{subarray}{c}{\text { \& } \\
v\left(x_{k}-L, t\right)<\varepsilon{ k \in I _ { n } \\
\begin{subarray} { c } { \text { \& } \\
v ( x _ { k } - L , t ) < \varepsilon } }\end{subarray}}\left(\mu\left(x_{k}-L, t\right) \text { or } v\left(x_{k}-L, t\right)\right) \\
& \leqslant \frac{M}{\lambda_{n}} \mid\left\{k \in I_{n}: \mu\left(x_{k}-L, t\right) \leqslant 1-\varepsilon\right. \text { or } \\
& \left.v\left(x_{k}-L, t\right) \geqslant \varepsilon\right\} \mid+\varepsilon .
\end{aligned}
$$

Note that

$$
\begin{aligned}
A_{\mu, \nu}(\varepsilon, t)= & \left\{n \in \mathbb{N}: \frac{1}{\lambda_{n}} \mid\left\{k \in I_{n}: \mu\left(x_{k}-L, t\right)\right.\right. \\
& \left.\left.\leqslant 1-\varepsilon \text { or } v\left(x_{k}-L, t\right) \geqslant \varepsilon\right\} \mid \geqslant \frac{\varepsilon}{M}\right\}
\end{aligned}
$$


belong to $\mathscr{I}$. If $n \in\left(A_{\mu, \nu}(\varepsilon, t)\right)^{c}$ then we have

$$
\begin{aligned}
& \frac{1}{\lambda_{n}} \sum_{k \in I_{n}} \mu\left(x_{k}-L, t\right)>1-2 \varepsilon \text { or } \\
& \frac{1}{\lambda_{n}} \sum_{k \in I_{n}} v\left(x_{k}-L, t\right)<2 \varepsilon .
\end{aligned}
$$

Now

$$
\begin{aligned}
T_{\mu, \nu}(\varepsilon, t)= & \left\{n \in \mathbb{N}: \frac{1}{\lambda_{n}} \sum_{k \in I_{n}} \mu\left(x_{k}-L, t\right) \leqslant\right. \\
& \left.1-2 \varepsilon \text { or } \frac{1}{\lambda_{n}} \sum_{k \in I_{n}} v\left(x_{k}-L, t\right) \geqslant 2 \varepsilon\right\} .
\end{aligned}
$$

Hence $T_{\mu, v}(\varepsilon, t) \subset A_{\mu, v}(\varepsilon, t)$ and so, by definition of an ideal, $T_{\mu, \nu}(\varepsilon, t) \in \mathscr{I}$. Hence we conclude that $x_{k} \rightarrow L[V, \lambda]^{(\mu, v)}(\mathscr{I})$. (iii) This readily follows from (i) and (ii).

\section{Theorem 2}

(i) $S^{(\mu, v)}(\mathscr{I}) \subset S_{\lambda}^{(\mu, v)}(\mathscr{I})$ if $\liminf _{n \rightarrow \infty} \lambda_{n} / n>0$.

(ii) If lim inf ${ }_{n \rightarrow \infty} \lambda_{n} / n=0, \mathscr{I}$-strongly (by which we mean that $\exists$ a subsequence $(n(j))_{j=1}^{\infty}$, for which $\left(\lambda_{n(j)} / n(j)\right)(1 / j) \forall j$ and $\left.\{n(j): j \in \mathbb{N}\} \notin \mathscr{I}\right)$ then $S^{(\mu, v)}(\mathscr{I}) \varsubsetneqq S_{\lambda}^{(\mu, v)}(\mathscr{I})$.

Proof: (i) For given $\varepsilon>0$ and every $t>0$, we have

$$
\begin{aligned}
\frac{1}{n} \mid\left\{k \leqslant n: \mu\left(x_{k}-L, t\right) \leqslant 1-\varepsilon\right. \text { or } \\
\\
\left.v\left(x_{k}-L, t\right) \geqslant \varepsilon\right\} \mid \\
\geqslant \frac{1}{n} \mid\left\{k \in I_{n}: \mu\left(x_{k}-L, t\right) \leqslant 1-\varepsilon\right. \text { or } \\
\left.v\left(x_{k}-L, t\right) \geqslant \varepsilon\right\} \mid \\
=\frac{\lambda_{n}}{n} \frac{1}{\lambda_{n}} \mid\left\{k \in I_{n}: \mu\left(x_{k}-L, t\right) \leqslant 1-\varepsilon\right. \\
\left.\quad \text { or } v\left(x_{k}-L, t\right) \geqslant \varepsilon\right\} \mid .
\end{aligned}
$$

If $\liminf _{n \rightarrow \infty} \lambda_{n} / n=\alpha$ then from the definition $\{n \in$ $\left.\mathbb{N}: \lambda_{n} / n<\frac{1}{2} \alpha\right\}$ is finite. For every $\delta>0$,

$$
\begin{aligned}
&\left\{n \in \mathbb{N}: \frac{1}{\lambda_{n}} \mid\left\{k \in I_{n}: \mu\left(x_{k}-L, t\right) \leqslant 1-\varepsilon\right.\right. \\
&\text { or } \left.\left.v\left(x_{k}-L, t\right) \geqslant \varepsilon\right\} \mid \geqslant \delta\right\} \\
& \subset\left\{n \in \mathbb{N}: \frac{1}{n} \mid\left\{k \in I_{n}: \mu\left(x_{k}-L, t\right) \leqslant 1-\varepsilon\right.\right. \\
&\left.\left.\quad \text { or } v\left(x_{k}-L, t\right) \geqslant \varepsilon\right\} \mid \geqslant \frac{\alpha}{2} \delta\right\} \\
& \cup\left\{n \in \mathbb{N}: \frac{\lambda_{n}}{n}<\frac{\alpha}{2}\right\} .
\end{aligned}
$$

Since $\mathscr{I}$ is admissible, the set on the right-hand side belongs to $\mathscr{I}$ and this completed the proof of (i). (ii) The proof is standard.

Theorem 3 Let $(X, \mu, v, *, \diamond)$ be an IFNS. If $\lambda \in \Delta$ is such that $\lim _{n} \lambda_{n} / n=1$, then $S_{\lambda}^{(\mu, v)}(\mathscr{I}) \subset S^{(\mu, \nu)}(\mathscr{I})$.

Proof: Let $\delta>0$ be given. Since $\lim _{n} \lambda_{n} / n=1$, we can choose $m \in \mathbb{N}$ such that $\mu\left(\lambda_{n} / n-1, t\right)>1-\frac{1}{2} \delta$ or $v\left(\lambda_{n} / n-1, t\right)<\frac{1}{2} \delta$, for all $n \geqslant m$. Now observe that, for $\varepsilon>0$, every $t>0$ and $n \geqslant m$

$$
\begin{gathered}
\frac{1}{n} \mid\left\{k \leqslant n: \mu\left(x_{k}-L, t\right) \leqslant 1-\varepsilon \text { or } v\left(x_{k}-L, t\right) \geqslant \varepsilon\right\} \mid \\
=\frac{1}{n} \mid\left\{k \leqslant n-\lambda_{n}: \mu\left(x_{k}-L, t\right) \leqslant 1-\varepsilon\right. \text { or } \\
\left.v\left(x_{k}-L, t\right) \geqslant \varepsilon\right\} \mid \\
+\frac{1}{n} \mid\left\{k \in I_{n}: \mu\left(x_{k}-L, t\right) \leqslant 1-\varepsilon\right. \text { or } \\
\left.v\left(x_{k}-L, t\right) \geqslant \varepsilon\right\} \mid \\
\leqslant \frac{n-\lambda_{n}}{n}+\frac{1}{n} \mid\left\{k \in I_{n}: \mu\left(x_{k}-L, t\right) \leqslant 1-\varepsilon\right. \\
\left.\quad \text { or } v\left(x_{k}-L, t\right) \geqslant \varepsilon\right\} \mid \\
\leqslant 1-\left(1-\frac{\delta}{2}\right)+\frac{1}{n} \mid\left\{k \in I_{n}: \mu\left(x_{k}-L, t\right) \leqslant\right. \\
\left.1-\varepsilon \text { or } v\left(x_{k}-L, t\right) \geqslant \varepsilon\right\} \mid \\
=\frac{\delta}{2}+\frac{1}{n} \mid\left\{k \in I_{n}: \mu\left(x_{k}-L, t\right) \leqslant 1-\varepsilon\right. \\
\left.\quad \text { or } v\left(x_{k}-L, t\right) \geqslant \varepsilon\right\} \mid .
\end{gathered}
$$

Hence

$$
\begin{aligned}
& \left\{n \in \mathbb{N}: \frac{1}{n} \mid\left\{k \leqslant n: \mu\left(x_{k}-L, t\right) \leqslant 1-\varepsilon\right.\right. \\
& \left.\left.\quad \text { or } v\left(x_{k}-L, t\right) \geqslant \varepsilon\right\} \mid \geqslant \delta\right\} \\
& \subset\left\{n \in \mathbb{N}: \frac{1}{n} \mid\left\{k \in I_{n}: \mu\left(x_{k}-L, t\right) \leqslant 1-\varepsilon\right.\right. \\
& \left.\left.\quad \text { or } v\left(x_{k}-L, t\right) \geqslant \varepsilon\right\} \mid \geqslant \frac{\delta}{2}\right\} \cup\{1,2,3, \ldots, m\} .
\end{aligned}
$$

If $\mathscr{I}-S_{\lambda}^{(\mu, v)}-\lim x=L$ then the set on the right-hand side belongs to $\mathscr{I}$ and so the set on the left-hand side also belongs to $\mathscr{I}$. This shows that $x=\left(x_{k}\right)$ is $\mathscr{I}$-statistically convergent to $L$ with respect to the intuitionistic fuzzy norm $(\mu, v)$.

Theorem 4 Let $(X, \mu, v, *, \diamond)$ be an IFNS such that $\frac{1}{4} \varepsilon_{n} \diamond \frac{1}{4} \varepsilon_{n}<\frac{1}{2} \varepsilon_{n}$ and $\left(1-\frac{1}{4} \varepsilon_{n}\right) *\left(1-\frac{1}{4} \varepsilon_{n}\right)>1-\frac{1}{2} \varepsilon_{n}$. If $X$ is a Banach space then $S_{\lambda}^{(\mu, v)}(\mathscr{I}) \cap m(X)$ is a closed subset of $m(X)$ 
Proof: We first assume that $\left(x^{n}\right) \subset S_{\lambda}^{(\mu, v)}(\mathscr{I}) \cap m(X)$ is a convergent sequence and it converges to $x \in$ $m(X)$. We need to show that $x \in S_{\lambda}^{(\mu, v)}(\mathscr{I}) \cap m(X)$. Suppose that $x^{n} \rightarrow L_{n}\left(S_{\lambda}^{(\mu, \nu)}(\mathscr{I})\right)$ for all $n \in \mathbb{N}$. Take a sequence $\left\{\varepsilon_{n}\right\}_{n \in \mathbb{N}}$ of strictly decreasing positive numbers converging to zero. We can find an $n \in \mathbb{N}$ such that $\sup _{j} v\left(x-x^{j}, t\right)<\frac{1}{4} \varepsilon_{n}$ for all $j \geqslant n$. Choose $0<\delta<\frac{1}{5}$. Now let

$$
\begin{gathered}
A_{\mu, v}\left(\varepsilon_{n}, t\right)=\left\{m \in \mathbb{N}: \frac{1}{\lambda_{m}} \mid\left\{k \in I_{m}:\right.\right. \\
\mu\left(x_{k}^{n}-L_{n}, t\right) \leqslant 1-\frac{\varepsilon_{n}}{4} \text { or } \\
\left.\left.v\left(x_{k}^{n}-L_{n}, t\right) \geqslant \frac{\varepsilon_{n}}{4}\right\} \mid<\delta\right\}
\end{gathered}
$$

belongs to $F(\mathscr{I})$ and

$$
\begin{gathered}
B_{\mu, v}\left(\varepsilon_{n}, t\right)=\left\{m \in \mathbb{N}: \frac{1}{\lambda_{m}} \mid\left\{k \in I_{m}:\right.\right. \\
\mu\left(x_{k}^{n+1}-L_{n+1}, t\right) \leqslant 1-\frac{\varepsilon_{n}}{4} \text { or } \\
\left.\left.\nu\left(x_{k}^{n+1}-L_{n+1}, t\right) \geqslant \frac{\varepsilon_{n}}{4}\right\} \mid<\delta\right\}
\end{gathered}
$$

belongs to $F(\mathscr{I})$. Since $A_{\mu, v}\left(\varepsilon_{n}, t\right) \cap B_{\mu, v}\left(\varepsilon_{n}, t\right) \in$ $F(\mathscr{I})$ and $\varnothing \notin F(\mathscr{I})$, we can choose $m \in A_{\mu, v}\left(\varepsilon_{n}, t\right) \cap$ $B_{\mu, v}\left(\varepsilon_{n}, t\right)$. Then

$$
\begin{gathered}
\frac{1}{\lambda_{m}} \mid\left\{k \in I_{m}: \mu\left(x_{k}^{n}-L_{n}, t\right) \leqslant 1-\frac{\varepsilon_{n}}{4}\right. \\
\text { or } v\left(x_{k}^{n}-L_{n}, t\right) \geqslant \frac{\varepsilon_{n}}{4} \vee \\
\mu\left(x_{k}^{n+1}-L_{n+1}, t\right) \leqslant 1-\frac{\varepsilon_{n}}{4} \text { or } \\
\left.v\left(x_{k}^{n+1}-L_{n+1}, t\right) \geqslant \frac{\varepsilon_{n}}{4}\right\} \mid \leqslant 2 \delta<1 .
\end{gathered}
$$

Since $\lambda_{m} \rightarrow \infty$ and $A_{\mu, v}\left(\varepsilon_{n}, t\right) \cap B_{\mu, v}\left(\varepsilon_{n}, t\right) \in F(\mathscr{I})$ is infinite, we can choose the above $m$ so that $\lambda_{m}>5$. Hence there must exist a $k \in I_{m}$ for which we have simultaneously, $\mu\left(x_{k}^{n}-L_{n}, t\right)>1-\frac{1}{4} \varepsilon_{n}$ or $v\left(x_{k}^{n}-L_{n}, t\right)<\frac{1}{4} \varepsilon_{n}$ and $\mu\left(x_{k}^{n+1}-L_{n+1}, t\right)>1-\frac{1}{4} \varepsilon_{n}$ or $v\left(x_{k}^{n+1}-L_{n+1}, t\right)<\frac{1}{4} \varepsilon_{n}$. For a given $\varepsilon_{n}>0$ choose $\frac{1}{2} \varepsilon_{n}$ such that $\left(1-\frac{1}{2} \varepsilon_{n}\right) *\left(1-\frac{1}{2} \varepsilon_{n}\right)>1-\varepsilon_{n}$ and $\frac{1}{2} \varepsilon_{n} \diamond \frac{1}{2} \varepsilon_{n}<\varepsilon_{n}$. Then it follows that

$$
\begin{aligned}
& v\left(L_{n}-x_{k}^{n}, \frac{t}{2}\right) \diamond v\left(L_{n+1}-x_{k}^{n+1}, \frac{t}{2}\right) \\
& \quad \leqslant \frac{\varepsilon_{n}}{4} \diamond \frac{\varepsilon_{n}}{4}<\frac{\varepsilon_{n}}{2}
\end{aligned}
$$

and

$$
\begin{aligned}
v\left(x_{k}^{n}-x_{k}^{n+1}, t\right) & \leqslant \sup _{n} v\left(x-x^{n}, \frac{t}{2}\right) \\
& \diamond \sup _{n} v\left(x-x^{n+1}, \frac{t}{2}\right) \\
& \leqslant \frac{\varepsilon_{n}}{4} \diamond \frac{\varepsilon_{n}}{4}<\frac{\varepsilon_{n}}{2} .
\end{aligned}
$$

Hence we have

$$
\begin{aligned}
v\left(L_{n}-L_{n+1}, t\right) \leqslant & {\left[v\left(L_{n}-x_{k}^{n}, \frac{t}{3}\right)\right.} \\
& \left.\diamond v\left(x_{k}^{n+1}-L_{n+1}, \frac{t}{3}\right)\right] \\
& \diamond v\left(x_{k}^{n}-x_{k}^{n+1}, \frac{t}{3}\right) \\
\leqslant & \frac{\varepsilon_{n}}{2} \diamond \frac{\varepsilon_{n}}{2}<\varepsilon_{n}
\end{aligned}
$$

and similarly $\mu\left(L_{n}-L_{n+1}, t\right)>1-\varepsilon_{n}$. This implies that $\left\{L_{n}\right\}_{n \in \mathbb{N}}$ is a Cauchy sequence in $X$ and let $L_{n} \rightarrow L \in X$ as $n \rightarrow \infty$. We shall prove that $x \rightarrow$ $L\left(S_{\lambda}^{(\mu, v)}(\mathscr{I})\right)$. For any $\varepsilon>0$ and $t>0$, choose $n \in \mathbb{N}$ such that $\varepsilon_{n}<\frac{1}{4} \varepsilon, \sup _{n} v\left(x-x^{n}, t\right)<\frac{1}{4} \varepsilon$, $\mu\left(L_{n}-L, t\right)>1-\frac{1}{4} \varepsilon$ or $v\left(L_{n}-L, t\right)<\frac{1}{4} \varepsilon$. Now since

$$
\begin{aligned}
& \frac{1}{\lambda_{n}}\left|\left\{k \in I_{n}: v\left(x_{k}-L, t\right) \geqslant \varepsilon\right\}\right| \\
& \leqslant \frac{1}{\lambda_{n}} \mid\left\{k \in I_{n}: v\left(x_{k}-x_{k}^{n}, \frac{t}{3}\right) \diamond\right. \\
& \left.\quad\left[v\left(x_{k}^{n}-L_{n}, \frac{t}{3}\right) \diamond v\left(L_{n}-L, \frac{t}{3}\right)\right] \geqslant \varepsilon\right\} \mid \\
& \leqslant \frac{1}{\lambda_{n}}\left|\left\{k \in I_{n}: v\left(x_{k}^{n}-L_{n}, \frac{t}{3}\right) \geqslant \frac{\varepsilon}{2}\right\}\right|
\end{aligned}
$$

and similarly

$$
\begin{aligned}
\frac{1}{\lambda_{n}} \mid\{k & \left.\in I_{n}: \mu\left(x_{k}-L, t\right) \leqslant 1-\varepsilon\right\} \mid \\
> & \frac{1}{\lambda_{n}}\left|\left\{k \in I_{n}: \mu\left(x_{k}^{n}-L, \frac{t}{3}\right) \leqslant 1-\frac{\varepsilon}{2}\right\}\right| .
\end{aligned}
$$

It follows that

$$
\begin{gathered}
\left\{n \in \mathbb{N}: \frac{1}{\lambda_{n}} \mid\left\{k \in I_{n}: \mu\left(x_{k}-L, t\right) \leqslant 1-\varepsilon\right.\right. \\
\left.\left.\quad \text { or } v\left(x_{k}-L, t\right) \geqslant \varepsilon\right\} \mid \geqslant \delta\right\} \\
\subset\left\{n \in \mathbb{N}: \frac{1}{\lambda_{n}} \mid\left\{k \in I_{n}: \mu\left(x_{k}^{n}-L, \frac{t}{3}\right) \leqslant\right.\right. \\
\left.\left.1-\frac{\varepsilon}{2} \text { or } v\left(x_{k}^{n}-L, \frac{t}{3}\right) \geqslant \frac{\varepsilon}{2}\right\} \mid \geqslant \delta\right\}
\end{gathered}
$$

for any given $\delta>0$. Hence we have $x \rightarrow$ $L\left(S_{\lambda}^{(\mu, v)}(\mathscr{I})\right)$. 


\section{CONCLUSIONS}

In this paper we introduce the notions of $\mathscr{I}-[V, \lambda]$ summability and $\mathscr{I}$ - $\lambda$-statistical convergence with respect to the intuitionistic fuzzy norm $(\mu, v)$, investigate their relationship, and make some observations about these classes. We intend to unify these two approaches and use ideals to introduce the concept of $\mathscr{I}-\lambda$-statistical convergence with respect to the intuitionistic fuzzy norm $(\mu, v)$. Our study of $\mathscr{I}$-statistical and $\mathscr{I}$ - $\lambda$-statistical convergence convergence of sequences in intuitionistic fuzzy normed spaces also provides a tool to deal with convergence problems of sequences of fuzzy real numbers. These results can be used to study the convergence problems of sequences of fuzzy numbers having a chaotic pattern in intuitionistic fuzzy normed spaces.

Acknowledgements: The authors thank the referees for their comments.

\section{REFERENCES}

1. Fast H (1951) Sur la convergence statistique. Colloq Math 2, 241-4.

2. Fridy JA (1985) On statistical convergence. Analysis 5, 301-13.

3. Šalát T (1980) On statistically convergent sequences of real numbers. Math Slovaca 30, 139-50.

4. Kostyrko P, Šalát T, Wilczynki W (2000-2001) $\mathscr{I}$ convergence. Real Anal Exchange 26, 669-85.

5. Das P, Ghosal S (2010) Some further results on $\mathscr{I}$ Cauchy sequences and condition (AP). Comput Math Appl 59, 2597-600

6. Das P, Savaş E, Ghosal SKr (2011) On generalizations of certain summability methods using ideals. Appl Math Lett 24, 1509-14.

7. Savaş E, Das P (2011) A generalized statistical convergence via ideals. Appl Math Lett 24, 826-30.

8. Mursaleen M (2000) $\lambda$-statistical convergence. Math Slovaca 50, 111-5.

9. Leindler L (1965) Über die verallgemeinerte de la Vallée-Poussinsche summierbarkeit allgemeiner Orthogonalreihen. Acta Math Acad Sci Hungar 16, 375-87.

10. Kolk E (1991) The statistical convergence in Banach spaces. Acta Comment Univ Tartu 928, 41-52.

11. Zadeh LA (1965) Fuzzy sets. Inform Contr 8, 338-53.

12. Atanassov KT (1986) Intuitionistic fuzzy sets. Fuzzy Set Syst 20, 87-96.

13. Atanassov K, Pasi G, Yager R (2002) Intuitionistic fuzzy interpretations of multi-person multicriteria decision making. Proceedings of the 1st International IEEE Symposium on Intelligent Systems 1, 115-9.

14. Park JH (2004) Intuitionistic fuzzy metric spaces. Chaos Soliton Fract 22, 1039-46.
15. Saadati R, Park JH (2006) On the intuitionistic fuzzy topological spaces. Chaos Soliton Fract 27, 331-44.

16. Debnath P (2012) Lacunary ideal convergence in intuitionistic fuzzy normed linear spaces. Comput Math Appl 63, 708-15.

17. Debnath P, Sen M (2014) Some completeness results in terms of infinite series and quotient spaces in intuitionistic fuzzy $n$-normed linear spaces. $J$ Intell Fuzzy Syst 26, 975-82.

18. Debnath P, Sen M (2014) Some results of calculus for functions having values in an intuitionistic fuzzy $n$ normed linear space. J Intell Fuzzy Syst 26, 2983-91.

19. Debnath P (2015) Results on lacunary difference ideal convergence in intuitionistic fuzzy normed linear spaces. J Intell Fuzzy Syst 28, 1299-306.

20. Schweizer B, Sklar A (1960) Statistical metric spaces. Pac J Math 10, 313-34.

21. Savaş E, Gürdal M (2014) Certain summability methods in intuitionistic fuzzy normed spaces. $J$ Intell Fuzzy Syst 27, 1621-9.

22. Karakus S, Demirci K, Duman O (2008) Statistical convergence on intuitionistic fuzzy normed spaces. Chaos Soliton Fract 35, 763-9. 